Article

\title{
Optical Diagnostic Characterization of the Local Arc on Contaminated Insulation Surface at Low Pressure ${ }^{\dagger}$
}

\author{
Hao Yang ${ }^{1, *(\mathbb{D}}$, Haotian Zhang ${ }^{1}$, Wen Cao ${ }^{1}$, Xuanxiang Zhao ${ }^{1}$, Ran Wen ${ }^{1}$, Junping Zhao ${ }^{2}$, Shengwu Tan ${ }^{3}$ \\ and Pengchao Wang ${ }^{3}$
}

1 School of Electronics and Information, Xi'an Polytechnic University, Xi'an 710048, China; haotianzhang163@163.com (H.Z.); caowen@xpu.edu.cn (W.C.); xuanxiangzhao@163.com (X.Z.); wenran1994@126.com (R.W.)

2 School of Electrical Engineering, Xi'an Jiaotong University, Xi'an 710049, China; junping_zhao@mail.xjtu.edu.cn

3 Pinggao Group Company Limited, Pingdingshan 467000, China; shengwutan@163.com (S.T.); wangpengchao188@163.com (P.W.)

* Correspondence: yanghao@xpu.edu.cn

$+\quad$ This paper is an extended version of our paper published in 2020 IEEE International Conference on High Voltage Engineering and Application (ICHVE), Beijing, China, 6-10 September 2020; pp. 1-4.

Citation: Yang, H.; Zhang, H.; Cao, W.; Zhao, X.; Wen, R.; Zhao, J.; Tan, S.; Wang, P. Optical Diagnostic

Characterization of the Local Arc on Contaminated Insulation Surface at Low Pressure. Energies 2021, 14, 6116. https://doi.org/10.3390/en14196116

Academic Editors: Issouf Fofana and Bo Zhang

Received: 17 August 2021

Accepted: 21 September 2021

Published: 26 September 2021

Publisher's Note: MDPI stays neutral with regard to jurisdictional claims in published maps and institutional affiliations.

Copyright: (c) 2021 by the authors. Licensee MDPI, Basel, Switzerland. This article is an open access article distributed under the terms and conditions of the Creative Commons Attribution (CC BY) license (https:// creativecommons.org/licenses/by/ $4.0 /)$.

\begin{abstract}
Flashover of contaminated insulators is a major problem for power systems at high altitude. Laboratory experiments have shown that the optical diagnostic method can provide extensive information on the physical process of contamination flashover. In this paper, a study of the local arc on a wet polluted surface under low pressure by using the optical diagnostic method is presented. The thickness of the continuous spectrum, spectral line intensity and the spectral composition varies significantly in different stages of the local arc development. Thermodynamic parameters of the local arc (including electron temperature, electron density and conductivity) are obtained by analyzing the spectra. Both the electron temperature and the conductivity increase with the increase in leakage current and air pressure. Although the electron density does not change significantly with an increase in leakage current, it increases significantly with an increase in air pressure. The findings of this work could be used as supplementary information for the investigation of local arc parameters, thus providing a reliable reference for the calculation of contamination flashover at high altitude.
\end{abstract}

Keywords: contamination flashover; the local arc; low pressure; optical diagnostic method

\section{Introduction}

Line insulators are some of the most widely used components in transmission lines, and their performance directly affects the safe operation of power systems. When contamination that has accumulated on the surface of the insulators contains a conductive medium, it might cause a contamination flashover accident in the fog and drizzle [1-3]. The contamination status of insulators and the climate circumstance are very close in the same area $[4,5]$. When contamination flashover occurs on a single insulator string, other insulators in this area are also on the edge of flashover [6]. It is known that contamination flashover of insulators often causes massive blackouts, resulting in huge economic losses [7-9].

Contamination flashover of outdoor insulators remains a major problem for transmission lines [10,11]. Especially in high-altitude areas, the contamination flashover voltage of the line insulators is greatly reduced due to the lower air pressure [12,13]. Additionally, transmission lines inevitably have to pass through high-altitude areas $[14,15]$. Therefore, the study on the flashover process on the insulator surface under low pressure is of great significance for predicting the flashover voltage and preventing contamination flashover accidents. 
Contamination flashover is the process in which the local arc develops on the surface of the insulator, until the high-voltage electrode and ground electrode are connected $[16,17]$. Hence, it is helpful to understand the physical process by obtaining the thermodynamic parameters of the local arc. Until now, former researchers mainly focused on the electrical parameters of the local arc. However, as a special kind of plasma, the local arc is mainly composed of free electrons and charged ions and has special properties quite different from resistance.

The thermodynamic parameters that characterize plasma mainly include electron temperature, excitation temperature, rotation temperature, electron density, thermal conductivity and electrical conductivity. It has been found that these parameters of the local arc play a very important role in the calculation of contamination flashover. M. Slama, from the Ampère Laboratory of the Central University of Technology, proposed a dynamic mathematical equation describing the relationship between the resistivity of the local arc channel and the arc temperature $[18,19]$. Based on the law of conservation of energy, W. Sima, from Chongqing University, obtained the mathematical expressions of the contamination flashover voltage and arc temperature [20]. It can be concluded that thermodynamic parameters of the local arc play a very important role in calculating the arc characteristics and predicting the flashover voltage.

However, there are relatively less experimental studies on the measurement of thermodynamic parameters of the local arc. A. Nekahi, from the University of Quebec, used fiber optic spectrometers to obtain the emission spectra of AC and DC of the local arcs on iced surfaces. By fitting the three molecular spectra of $\mathrm{OH}(\mathrm{A}-\mathrm{X}), \mathrm{NH}(\mathrm{A}-\mathrm{X})$ and $\mathrm{N}^{2+}$ $(\mathrm{B}-\mathrm{X})$, the relationship between the rotation temperature of the local arc and the leakage current was obtained, and the results show that the local arc rotation temperature increases with the increase in the leakage current amplitude [21,22]. S. Li of Tsinghua University also designed a spectral temperature measurement system to obtain the emission spectrum of the local arc in the critical flashover stage [23]. However, there is a lack of research on the thermodynamic parameters of the local arc on the wet contaminated insulation surface under low pressure.

In this paper, an optical diagnostic system is designed for investigating the characteristics of the local arc on the polluted surface under low pressure. A special tracing element is employed to obtain the spectrum of the local arc. Typical parameters including electron temperature, electron density and conductivity are obtained by analyzing the spectra of the local arc. This work can enrich the investigation of the local arc under low pressure and may provide useful parameters for the calculation of flashover voltage.

\section{Experimental Arrangements and Methods}

\subsection{Test Facilities}

In this paper, an optical diagnostic platform of the local arc under low pressure was established, as shown in Figure 1. The experiments were carried out in the QD660 artificial climate room of $\mathrm{Xi}^{\prime}$ an Jiaotong University. The climate chamber had a cylindrical structure with a diameter of $4 \mathrm{~m}$ and a height of $5.2 \mathrm{~m}$. Its size met the requirements of the artificial contamination flashover test for insulator strings of AC $110 \mathrm{kV}$ and below. The artificial climate chamber could be used to achieve any environmental parameter between $-50{ }^{\circ} \mathrm{C}+60^{\circ} \mathrm{C}$ and $0.05 \mathrm{MPa} \sim 0.1 \mathrm{MPa}$. The power was provided by a $160 \mathrm{kV} / 4 \mathrm{~A}, 600 \mathrm{kVA}$ transformer with a short-circuit impedance less than $5 \%$. The leakage current was measured by a power-frequency Rogowski coil.

The spraying system consisted of high-pressure water pumps, pipes and atomizing nozzles. The deionized water was pressurized by a high-pressure water pump, and sent through a pipeline to atomization nozzles, installed at multiple locations around the wall of the artificial climate chamber. By changing the size of the nozzle, the diameter of the droplets ranging from 15 to $2000 \mu \mathrm{m}$ could be obtained. The polluted specimen was installed horizontally in the center of the chamber, and the spraying system was then used to wet the specimen before the test. 


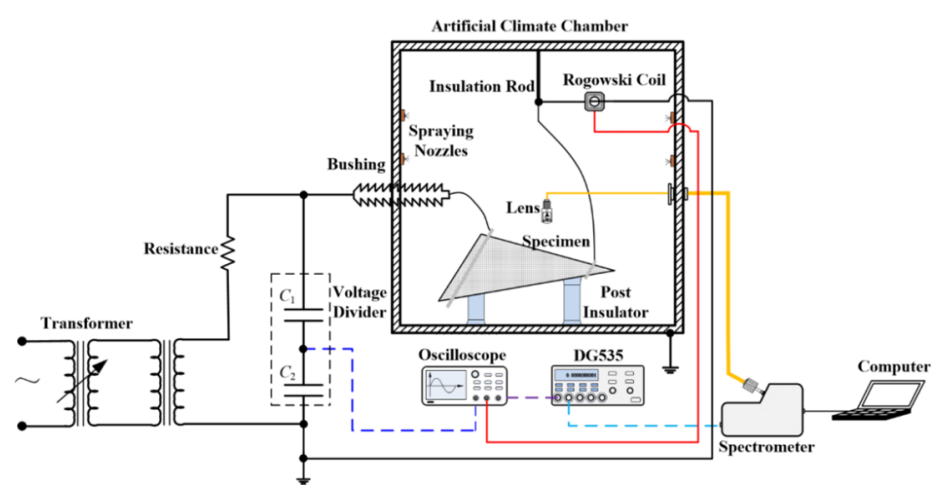

Figure 1. Spectrum diagnostic platform.

The spectrometer was used to record the spectra of the local arc. The wavelength range of the optical fiber spectrometer was $250-880 \mathrm{~nm}$, which meets the spectral measurement requirements. In order to increase the light intensity coupled into the spectrometer, a fiber collimator with a diameter of $45 \mathrm{~mm}$ was used to convert the arc radiation light into parallel light, and then the parallel light was coupled into the fiber through a light collector, as shown in Figure 2. A four-channel digital delay pulse signal generator (DG535) was used to trigger the spectrometer to acquire waveform and spectra synchronously.

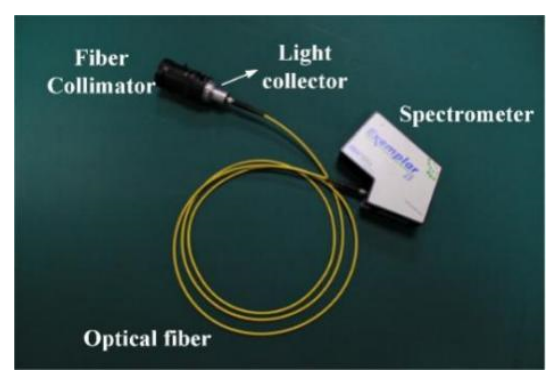

Figure 2. Spectral acquisition device.

\subsection{Specimen and Test Methods}

The development of the local arc over the insulator was random, resulting in low efficiency and poor repeatability of experimental measurement. Hence, a triangle glass plate was employed in our experiment, as shown in Figure 3a. The electric field intensity around the top corner of the triangular glass plate surface was higher than the rest of the region, as shown in Figure 3b, from our previous study. The local arc developed from the ground electrode and gradually developed to the high-voltage electrode. The glass triangular plate was polluted with a 2-cm narrow band for convenient measurement.

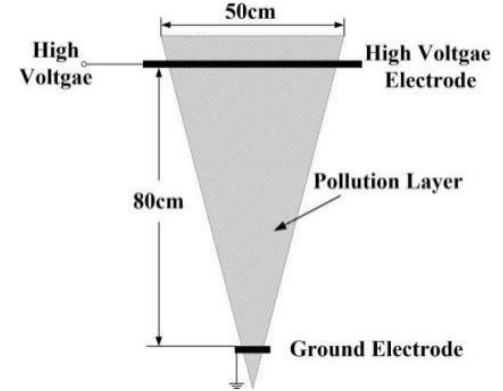

(a)

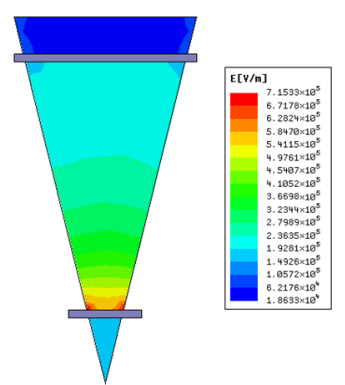

(b)

Figure 3. Triangular glass plate and its surface electric field distribution, (a) triangular glass plate used as test sample, (b) simulation results of the electric field distribution of the triangular glass surface. 
The solid layer method was used to pollute the specimens artificially, according to IEC 60,805 [24]. The artificial contamination was a mixture of sodium chloride, kaolin and de-ionized water. The equivalent salt deposit density (ESDD) was chosen as $0.1 \mathrm{mg} / \mathrm{cm}^{2}$, and the non-soluble deposit density (NSDD) was chosen as $0.1 \mathrm{mg} / \mathrm{cm}^{2}$. In the initial stage of the local arc, only a single spectral line could be found for the same element, i.e., Na I and K I, which did not meet the requirement of the spectral line relative intensity method, which requires "at least two atomic spectrum lines of the same element". Therefore, under the premise of not affecting the characteristics of the arc discharge, a trace amount of lithium was added as a tracer element to the artificial pollution for the occurrence of the useful spectral lines.

\subsection{Calculation of Plasma Parameters}

(1) Electron temperature

The electron temperature $\left(T_{e}\right)$ of the local arc can not only characterize the state of the particles in the plasma, but also be used to calculate the conductivity of the local arc. In this paper, $T_{e}$ of the local arc was calculated based on the spectral line relative intensity method. Using the two different excited state parameters of the same atom, $s$ and $t$, the formula for $T_{e}$ is obtained:

$$
T_{e}=\frac{-5040\left(E_{s}-E_{t}\right)}{\log (\lambda I / g A)_{s}-\log (\lambda I / g A)_{t}}
$$

In the formula, $E_{s}$ and $E_{t}$ are the energy of two different excited states of $s$ and $t$. Taking the atomic excitation energy as the independent variable and the formula $\log (\lambda I / g A)$ as the dependent variable, the Boltzmann diagram is obtained. The relationship between the slope $k$ of the straight line in the Boltzmann diagram and $T_{e}$ is:

$$
T_{e}=-\frac{5040}{k}
$$

In order to ensure high accuracy of the results, the energy level difference between the two spectral lines should be as large as possible, usually greater than $2 \mathrm{eV}$. The difference in excitation energy between the two spectral lines of $\mathrm{Li}$ I $610.4 \mathrm{~nm}$ and $\mathrm{Li} \mathrm{I} 670.8 \mathrm{~nm}$ is $2.63 \mathrm{eV}$, which meets the requirements of the spectral line relative intensity method. Therefore, Li I $610.4 \mathrm{~nm}$ and $\mathrm{Li}$ I $670.8 \mathrm{~nm}$ were chosen as the characteristic spectral lines, and Boltzmann's diagram was employed to calculate the $T_{e}$ of the local arc.

(2) Electron density

Electron density $\left(n_{e}\right)$ measurement methods include the Stark broadening method, Saha equation method, continuous spectroscopy method, Inglis-Teller method, etc. This paper chose the Stark broadening method with its high accuracy and simple measurement method to measure $n_{e}$. The relationship between the broadening value of the spectral line $\Delta \lambda$ and $n_{e}$ can be expressed as:

$$
\Delta \lambda=w n_{e}+1.75 \alpha n_{e}^{1 / 4}(1-1.2 \rho) w
$$

where $w$ is the Stark stretching factor; $\rho$ is the Debye shielding factor; and $\alpha$ is the broadening factor. According to reference [25], the equation above can be changed into:

$$
n_{e}=\frac{\Delta \lambda_{\text {NaStarkFWHM }}}{4.854} T^{0.126}
$$

where $\Delta \lambda_{\text {NaStarkFWHM }}$ is the width at half maximum (FWHM) of the Na I $568.8 \mathrm{~nm}$ spectral line, broadened by the Stark effect in nanometres. 


\section{(3) Electric conductivity}

Because there are a large number of electrons and ions in the arc plasma, it has a certain electrical conductivity $\left(\sigma_{s}\right)$. The Spitzer formula is not suitable to calculate the conductivity of low-temperature plasma. R. Mohanti and J. Gilligan, from North Carolina State University, conducted theoretical research on the conductivity of low-temperature plasma and modified the Spitzer formula [26]. The conductivity $\sigma_{s}$ can be calculated with $T_{e}$ and $n_{e}$, as shown in our former research [27]:

$$
\sigma_{s}=\frac{3.1 \times 10^{-2} T_{e}^{3 / 2}}{\ln \left(1+2.2 \times 10^{14} T_{e}^{3} / n_{e}\right)}
$$

\section{Typical Emission Spectrograms of the Local Arc}

The flashover development process can be divided into four stages: initiation, development, critical flashover and flashover. Assuming that the creepage distance is $L$, in the initial stage, the local arc length is shorter than $1 / 10 \mathrm{~L}$ and the leakage current amplitude $\left(i_{m}\right)$ is less than $50 \mathrm{~mA}$. In the development stage, the local arc length is between $1 / 10$ and $1 / 2 L$ and $i_{m}$ is between 50-150 mA. During the critical flashover stage, the local arc length is between $1 / 2$ and $2 / 3 L$ and $i_{m}$ is between 200 and $300 \mathrm{~mA}$. The optical fiber spectrometer was set to continuous acquisition mode to acquire the emission spectrum of the local arc from the initial stage to the flashover occurrence. The time-resolved emission spectrum of the local arc was obtained, as shown in Figure 4.

(1) The initial stage

In the initial stage of the local arc, $i_{m}$ is less than $50 \mathrm{~mA}$. In the spectrum shown in Figure 4a, no continuous spectral components can be observed; only the linear spectral lines of lithium, sodium and potassium atoms with a low relative intensity value can be found.

\section{(2) Development stage}

As $i_{m}$ continues to increase, the magnitude of the radiation spectrum line increases significantly. The energy injected into the arc channel continues to increase, and the collision between electrons and atoms becomes more intense, leading to the appearance of the atomic line of nitrogen in the spectrum, as shown in Figure $4 \mathrm{~b}$.

\section{(3) Critical flashover stage}

In the critical flashover stage, the copper atoms participate in the arc discharge, and three copper atomic lines $\mathrm{Cu} 510.5 \mathrm{~nm}, 515.8 \mathrm{~nm}$ and $521.8 \mathrm{~nm}$ appear in the spectrum. The dramatic increment in injection energy in the arc channel broadens the continuous spectrum thickness in the radiation spectrum, which leads to the increase in the ionization degree of particles in the channel, especially the alkali metal particles. Therefore, the K II $397.3 \mathrm{~nm}$ line spectrum was detected in the line, as shown in Figure 4c.

\section{(4) Flashover stage}

After the occurrence of flashover, the arc channel penetrates between the high-voltage electrode and the ground electrode, resulting in the energy of the power supply injecting into the arc channel almost instantaneously. The enhancement of the collisions between electrons and atoms/ions in this channel accelerate the ionization process of oxygen and nitrogen molecules. Therefore, oxygen ion line O II $485.7 \mathrm{~nm}$ and nitrogen ion line N II began to appear in the spectrum, as shown in Figure $4 \mathrm{~d}$. 


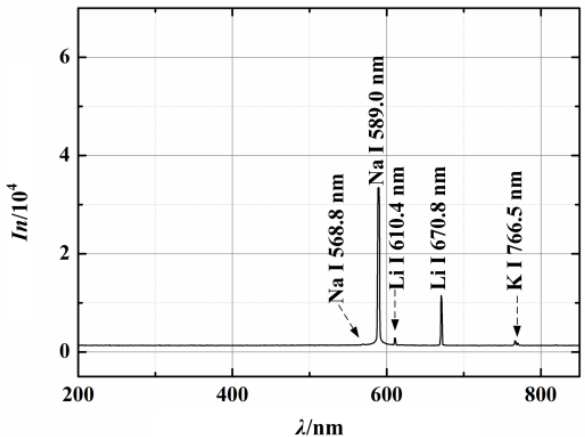

(a)

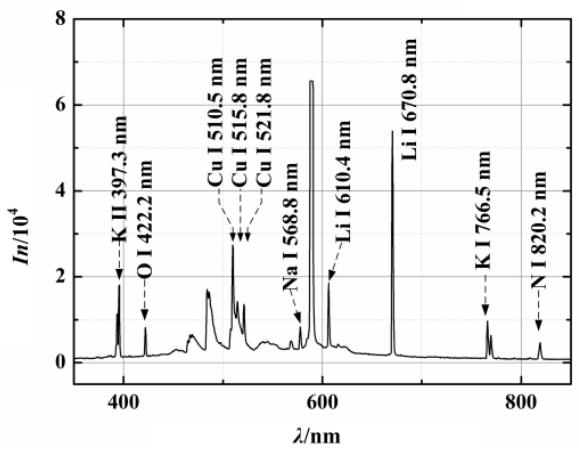

(c)

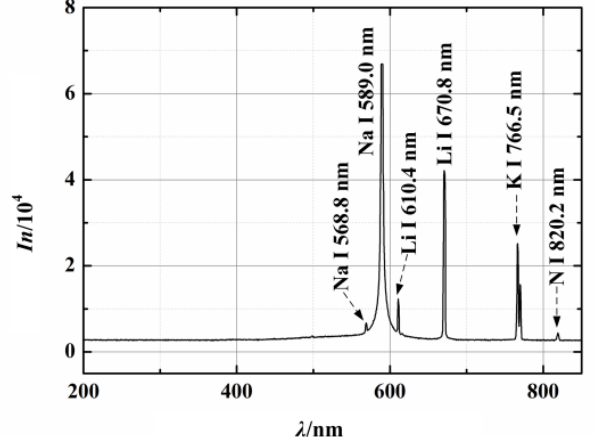

(b)

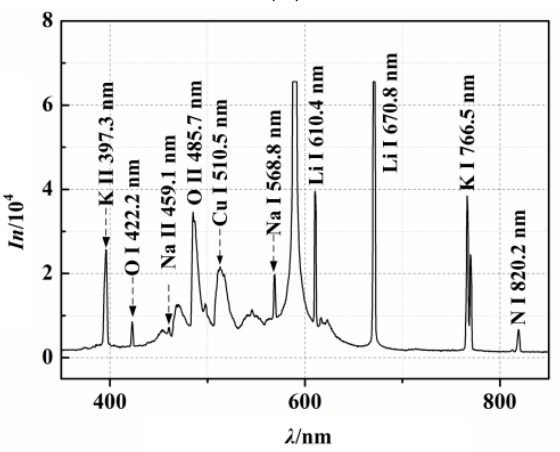

(d)

Figure 4. Typical spectrum of the local arc in different stages, (a) spectrum of local arc in initial stage, (b) spectrum of local arc in development stage, (c) spectrum of local arc in critical flashover stage,

(d) spectrum of local arc in flashover stage.

\section{Parameters of the Local Arc under Low Pressures}

Spectral diagnosis of the local arc on a wet contaminated insulation surface under low pressure was carried out in this section. Several vital parameters, such as electron temperature, electron density and conductivity were obtained.

\subsection{Electron Temperature of the Local Arc}

According to the actual altitude of the transmission line, four typical low-pressure values of $0.06,0.07,0.08$ and $0.09 \mathrm{MPa}$ were selected for the experiment in this paper. The experimental results show that the emission spectra of the local arc under different pressures are almost the same, similar to Figure 4. However, there is a significant difference in the ratio of the relative intensity of the discrete spectral lines. Li I $610.4 \mathrm{~nm}$ and Li I $670.8 \mathrm{~nm}$ were chosen as the characteristic spectral lines. The Boltzmann diagram method, mentioned in Section 2.3, was used to calculate the local arc electron temperature. The Boltzmann plots of the local arc emission spectra under different air pressures at $0.1 \mathrm{~A}$ are shown in Figure 5. The slope $k$ of the straight lines can be obtained for Equation (2) to calculate $T_{e}$.

Because the statics of $T_{e}$ show a large dispersion, the mathematical expectation value of the data was used to represent $T_{e}$ at a specific leakage current peak. $T_{e}$ of the local arc under different pressure is shown in Figure 6. 


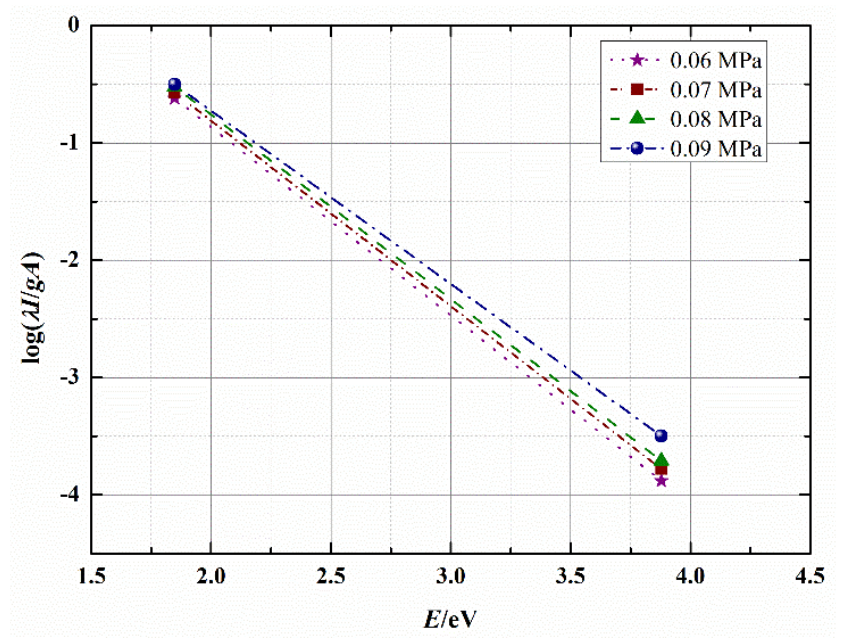

Figure 5. Boltzmann plots of the local arc emission spectra under different air pressures at $0.1 \mathrm{~A}$.

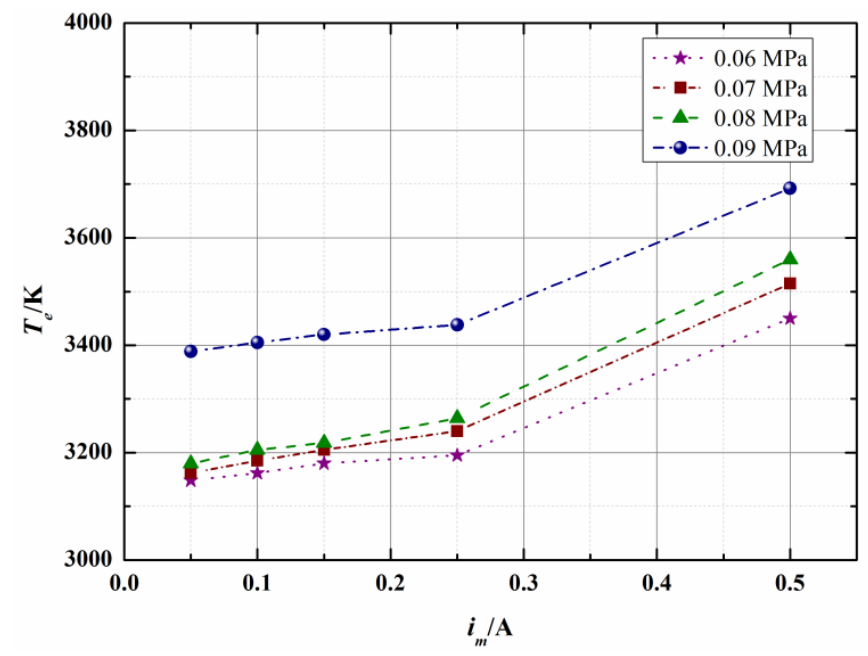

Figure 6. The value of $T_{\mathrm{e}}$ at different air pressures.

It can be seen from the figure that when the air pressure is $0.06 \mathrm{MPa}, T_{e}$ is between $3150 \mathrm{~K}$ and $3450 \mathrm{~K}$, and when the air pressure is $0.09 \mathrm{MPa}, T_{e}$ rises to the range between $3400 \mathrm{~K}$ and $3700 \mathrm{~K}$. It can be concluded that $T_{e}$ increases with the increase in $i_{m}$ at a certain pressure. When $i_{m}$ increases, the injection energy of the arc channel also increases, resulting in the enhancement of collisions between electrons and atoms/ions. Hence, $T_{e}$ increases rapidly with the increase in $i_{m}$.

Moreover, it can be concluded that $T_{e}$ increases with the increase in air pressure. Our previous research results show that the voltage gradient of local arc $(E)$ in a high-pressure environment is higher than that in a low-pressure environment when $i_{m}$ is the same [28]. The injection energy per unit length of the local arc in a high-pressure environment is relatively higher. In such cases, the movement of electrons in the arc channel intensifies due to higher energy. In other words, the migration speed of electrons increases. With the increase in air pressure, the number density of air molecules increases, resulting in the enhancement of inelastic collisions of electrons. Therefore, excitation and ionization collision frequencies are higher at a higher pressure, resulting in an increase in $T_{e}$.

\subsection{Electron Density of the Local Arc}

As mentioned above, the Stark broadening method was adopted to calculate $n_{e}$. In the critical flashover and flashover stages, the local arc radiation spectrum contains strong continuum components. In order to obtain the broadening value, the Lorentz linear 
function was used to perform the Na I $568.8 \mathrm{~nm}$ fitting. The value of $n_{e}$ under different air pressure is shown in Figure 7.

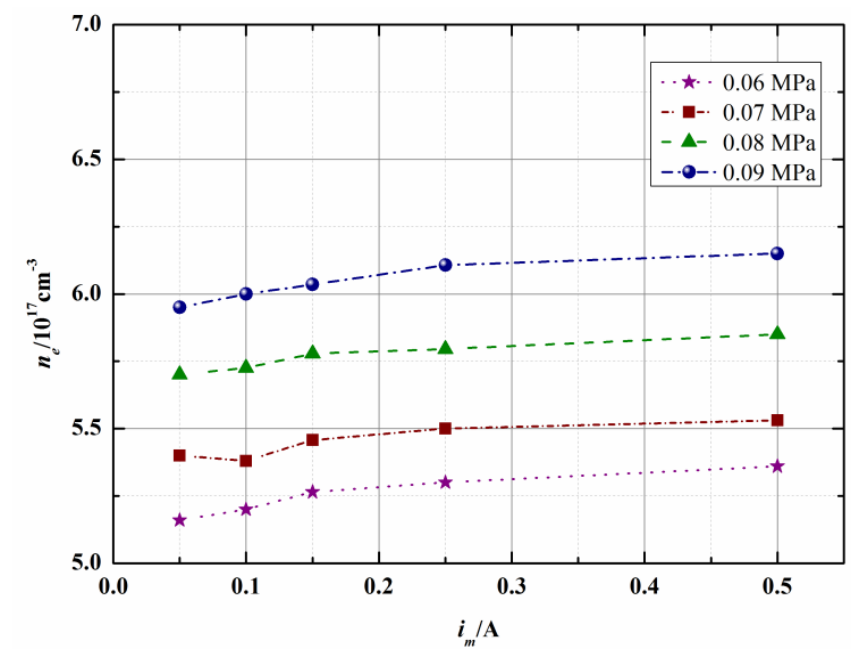

Figure 7. The value of $n_{\mathrm{e}}$ under different air pressures.

When $i_{m}$ is between $50 \mathrm{~mA}$ and $500 \mathrm{~mA}$, and the air pressure is between $0.06 \mathrm{MPa}$ and $0.09 \mathrm{MPa}$, the $n_{e}$ of the arc channel is between $5.2 \times 10^{17} \mathrm{~cm}^{-3}$ and $6.3 \times 10^{17} \mathrm{~cm}^{-3}$. With the increase in $i_{m}$, the ionization degree of gas particles increases, resulting in the increase in free electrons. In addition, the radius and volume of the local arc increase correspondingly. Therefore, $n_{e}$ does not change much during the development stage of the local arc. It can also be deduced that $n_{e}$ increases with the increase in the air pressure. When the outside air pressure increases, the energy of the arc channel increases and the local arc channel shrinks, leading to the increase in $n_{\mathrm{e}}$.

\subsection{Conductivity of the Local Arc}

Conductivity is one of the basic electrical parameters of the local arc, and it is also one of the critical parameters for calculating the voltage of the local arc. Compared with the "three-electrode" method [28], the non-contact spectroscopy method makes it much easier to obtain $\sigma_{s}$ of the local arc propagating over the polluted surface. The $\sigma_{s}$ of the arc channel can be calculated from Equation (5) by substituting $T_{\mathrm{e}}$ and $n_{\mathrm{e}}$ with the values in Figures 6 and 7. Figure 8 shows the relationship between $\sigma_{s}$ and $i_{m}$ under different pressure. Since the index value of $T_{e}$ is larger than $n_{e}$ in Equation (5), $\sigma_{s}$ and $T_{e}$ have the same changing trend.

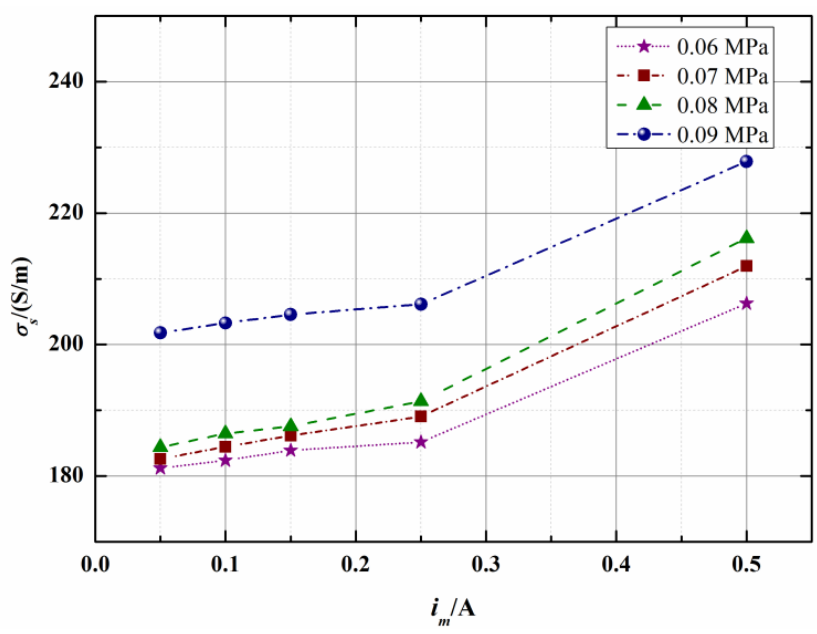

Figure 8. The value of $\sigma_{s}$ at different air pressures. 
Additionally, $\sigma_{s}$ can also be obtained by the calculation of electrical parameters of the local arc. The calculated results are compared with the spectral diagnosis results in the following part. In Rizk's former study, the relationship between $E$ and $i_{m}$ under low pressure was obtained, as shown in the following equation [29]:

$$
E=716\left(p / p_{0}\right)^{0.77} i_{m}^{-0.56}
$$

where $p$ is the actual pressure, $p_{0}$ is the ambient pressure. The relationship between $\sigma_{s}$ and $E$ can be expressed as follows:

$$
\sigma_{s}=\frac{i_{m}}{\pi E r^{2}}
$$

where $r$ is the arc boundary radius, and the relationship between $r$ and $p$ has been revealed by Rizk in Reference [29]:

$$
r \propto p^{-0.465}
$$

and the radius $r_{d}$ of the arc channel in ambient pressure is also a function of $i_{m}$ [30].

$$
r_{d}=\sqrt{\frac{i_{m}}{1.45 \pi}}
$$

Thus, we obtain:

$$
\sigma_{s}=\frac{i_{m}^{0.56}}{494\left(p / p_{0}\right)^{-0.16}}
$$

By solving Equation (10), $\sigma_{s}$ at a different pressure could be obtained, as shown in Figure 9. Compared with Figure 7, the calculated value of $\sigma_{s}$ is smaller. Since the plasma inside the local arc is not completely uniformly distributed, the true value of $r$ is not the arc boundary radius measured by actual experiments. The local arc channel is populated with high-density charged particles and the outer area of the channel is a heated and luminous area consisting of neutral atoms. It is generally believed that the maximum radial light intensity gradient of the channel is at the intersection of the ionization zone and the neutral atomic zone. That is, the radius corresponding to the maximum value is taken as the true radius value of the conductive area of the arc channel. Hence, the value of $r$ in Equation (7) is larger than the true value, resulting in a smaller value of $\sigma_{s}$. However, the deviations of $\sigma_{s}$ between the true value and the calculated value is trivial, within an order of magnitude. We can still consider our proposed approach of the spectral diagnostic method, which is relatively effective in measuring the local arc conductivity under low air pressure.

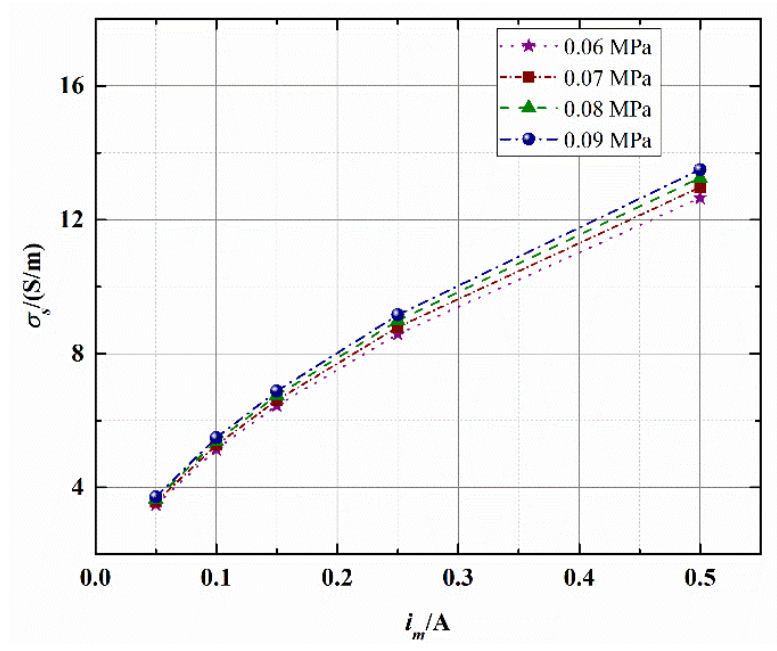

Figure 9. The calculated value of $\sigma_{s}$ at different air pressures. 


\section{Conclusions}

In this paper, we carried out the spectral diagnosis of the local arc on the wet contaminated insulation surface under low pressure. Key parameters of the local arc, including electron temperature, electron density and conductivity were calculated by spectroscopic measurements. The following conclusions were deduced:

(1) The spectra of the local arc are quite similar, even though the air pressure is different. The spectral characteristics of the different development stages of the local arc can be easily distinguished.

(2) Thermodynamic parameters of the local arc (including electron temperature and electron density) are obtained by analyzing the spectra. Electron temperature increases significantly with the increase in leakage current and air pressure, while, the electron density does not change much with the increase in leakage current, but it increases significantly with the increase in air pressure.

(3) The conductivity of the local arc is also calculated by employing the value of electron temperature and electron density in this paper. The conductivity increases with the increase in leakage current and air pressure. The deviation of conductivity between the true value and the calculated value is trivial.

Former researchers have used thermodynamic parameters of the local arc to predict the contamination flashover. In our further study, the obtained parameters of the local arc under low pressure will be used for the calculation of contamination flashover voltage of on-line insulators. This will be explored in our following paper.

Author Contributions: Conceptualization, H.Y.; Formal analysis, H.Y., W.C., X.Z., R.W. and J.Z.; Investigation, W.C., X.Z., R.W. and J.Z.; Writing—original draft, H.Z.; Writing-review and editing, S.T. and P.W. All authors have read and agreed to the published version of the manuscript.

Funding: This work was supported in part by the Science and Technology Project of State Grid Corporation of China (No. B491BG180016).

Institutional Review Board Statement: Not applicable.

Informed Consent Statement: Not applicable.

Data Availability Statement: Data sharing not applicable. No new data were created or analyzed in this study. Data sharing is not applicable to this article.

Conflicts of Interest: The authors declare no conflict of interest.

\section{References}

1. Tan, S.; Wang, P.; Zhao, J.; Yang, H.; Wen, R. Study on the Typical Optical Emission Spectrograms of AC Arc Discharge over the Polluted Insulation Surface. In Proceedings of the 2020 IEEE International Conference on High Voltage Engineering and Application (ICHVE), Beijing, China, 6-10 September 2020; pp. 1-4. [CrossRef]

2. Zhang, C.; Wang, L. Experimental Investigation on Pollution Flashover Performance of Multiple Parallel Suspension Insulators. IEEE Trans. Dielectr. Electr. Insul. 2016, 23, 2840-2849. [CrossRef]

3. Yang, Z.; Jiang, X.; Han, X.; Zhang, Z.; Hu, J. Influence of Pollution Chemical Components on Ac Flashover Performance of Various Types of Insulators. High Volt. 2019, 4, 105-112. [CrossRef]

4. He, J.; Gorur, R. Flashover of Insulators in a Wet Environment. IEEE Trans. Dielectr. Electr. Insul. 2017, 24, 1038-1044. [CrossRef]

5. Yang, L.; Zhang, F.; Wang, Z.; Jiang, X.; Hao, Y.; Li, L.; Liao, Y.; Zhang, F. Evaluation of Wetting Condition and Its Effects on contamination flashover Voltage of Aerodynamic Insulators. IEEE Trans. Dielectr. Electr. Insul. 2016, 23, 2875-2882. [CrossRef]

6. Shu, L.; Liu, Y.; Jiang, X.; Hu, Q.; He, G.; Yu, Z.; Xiao, L. Three-Dimensional Electric Field Simulation and Flashover Path Analysis of Ice-Covered Suspension Insulators. High Volt. 2020, 5, 327-333. [CrossRef]

7. Slama, M.E.A.; Albano, M.; Haddad, A.M.; Waters, R.T.; Cwikowski, O.; Iddrissu, I.; Knapper, J.; Scopes, O. Monitoring of Dry Bands and Discharge Activities at the Surface of Textured Insulators with AC Clean Fog Test Conditions. Energies 2021, 14, 2914. [CrossRef]

8. Shariatinasab, R.; Saghafi, S.; Khorashadizadeh, M.; Ghayedi, M. Probabilistic Assessment of Insulator Failure under Contaminated Conditions. IET Sci. Meas. Technol. 2020, 14, 557-563. [CrossRef]

9. Savadkoohi, E.M.; Mirzaie, M.; Seyyedbarzegar, S.; Mohammadi, M.; Khodsuz, M.; Pashakolae, M.G.; Ghadikolaei, M.B. Experimental Investigation on Composite Insulators AC Flashover Performance with Fan-Shaped Non-Uniform Pollution under Electro-Thermal Stress. Int. J. Electr. Power Energy Syst. 2020, 121, 106142. [CrossRef] 
10. Lan, L.; Zhang, G.; Wang, Y.; Wen, X.; Wang, W.; Pei, H. The Influence of Natural Contamination on Pollution Flashover Voltage Waveform of Porcelain Insulators in Heavily Polluted Area. IEEE Access 2019, 7, 121395-121406. [CrossRef]

11. Zhang, Z.; Zhang, W.; You, J.; Jiang, X.; Zhang, D.; Bi, M.; Wu, B.; Wu, J. Influence Factors in Contamination Process of XP-160 Insulators based on Computational Fluid Mechanics. IET Gener. Transm. Distrib. 2016, 10, 4140-4148. [CrossRef]

12. Qiao, X.; Zhang, Z.; Jiang, X.; Liang, T. Influence of DC Electric Fields on Pollution of HVDC Composite Insulator Short Samples with Different Environmental Parameters. Energies 2019, 12, 2304. [CrossRef]

13. Zhang, C.; Wang, L.; Guan, Z.; Zhang, F. Pollution Flashover Performance of Full-Scale \pm 800 KV Converter Station Post Insulators at High Altitude Area. IEEE Trans. Dielectr. Electr. Insul. 2013, 20, 717-726. [CrossRef]

14. Huang, D.; Ruan, J.; Cai, W.; Li, T.; Wei, Y.; Liu, J. Flashover Prevention on High-Altitude H Transmission Line Insulator Strings IEEE Trans. Dielectr. Electr. Insul. 2009, 16, 88-98. [CrossRef]

15. Rudakova, V.; Tikhodeev, N. Influence of Low Air Pressure on Flashover Voltages of Polluted Insulators: Test Data, Generalization Attempts and Some Recommendations. IEEE Trans. Power Deliv. 1989, 4, 607-613. [CrossRef]

16. Liao, Y.; Wang, Q.; Yang, L.; Kuang, Z.; Hao, Y.; Zhang, C. Discharge Behavior and Morphological Characteristics of Suspended Water-Drop on Shed Edge during Rain Flashover of Polluted Large-Diameter Post Insulator. Energies 2021, 14, 1652. [CrossRef]

17. Mei, H.; Guan, X.; Fu, X.; Zhao, C.; Wang, L. Influence of Tower Anticorrosion Coating as Contaminant on Operation Characteristics of Composite Insulator. High Volt. 2018, 3, 193-198. [CrossRef]

18. Michelarakis, M.; Widger, P.; Beroual, A.; Haddad, A. Electrical Detection of Creeping Discharges over Insulator Surfaces in Atmospheric Gases Under AC Voltage Application. Energies 2019, 12, 2970. [CrossRef]

19. Slama, M.E.A.; Beroual, A.; Hadi, H. Analytical Computation of Discharge Characteristic Constants and Critical Parameters of Flashover of Polluted Insulators. IEEE Trans. Dielectr. Electr. Insul. 2010, 17, 1764-1771. [CrossRef]

20. Sima, W.; Guo, F.; Yang, Q.; Yuan, T. Calculation of the Arc Velocity Along the Polluted Surface of Short Glass Plates Considering the Air Effect. Energies 2012, 5, 815-834. [CrossRef]

21. Nekahi, A.; Farokhi, S.; Farzaneh, M.; Stewart, B.G. Arc Energy and Temperature during Its Propagation Over Ice-Covered Surfaces. IEEE Trans. Plasma Sci. 2014, 42, 114-119. [CrossRef]

22. Nekahi, A.; Farzaneh, M. Excitation Temperature Determination of an Arc Formed over An Ice Surface Using Optical Emission Spectroscopy. IEEE Trans. Dielectr. Electr. Insul. 2011, 18, 1829-1834. [CrossRef]

23. Li, S.; Zhang, R.; Tan, K. Measurement of the Temperature of A the local arc Propagating along A Polluted Dielectric Surface. Proc. CSEE 1990, 10, 1-9. [CrossRef]

24. IEC. Artificial Pollution Tests on High-Voltage Ceramic and Glass Insulators to Be Used on A.C. Systems; IEC 60507; International Electrotechnical Commission: Geneva, Switzerland, 2013.

25. Griem, H.R. Principles of Plasma Spectroscopy; Cambridge University Press: Cambridge, UK, 1997; pp. $259-265$.

26. Zollweg, R.J.; Liebermann, R.W. Electrical Conductivity of Nonideal Plasmas. J. Appl. Phys. 1987, 62, 3621. [CrossRef]

27. Li, X.; Liu, X.; Zeng, F.; Yang, H.; Zhang, Q. Study on Resistance and Energy Deposition of Spark Channel under the Oscillatory Current Pulse. IEEE Trans. Plasma Sci. 2014, 42, 2259-2265. [CrossRef]

28. Yang, H.; Ren, Y.; Wen, R.; Zhang, L.; Chen, Y. Study on the time-varying characteristics of AC local arc over the contaminated insulation surface. AIP Adv. 2021, 11, 025028. [CrossRef]

29. Rizk, F.M.; Rezazada, A.Q. Modeling of Altitude Effects on Ac Flashover of Polluted High Voltage Insulators. IEEE Trans. Power Deliv. 1997, 12, 810-822. [CrossRef]

30. Wilkins, R. Flashover voltage of high-voltage insulators with uniform surface-pollution films. Proc. Inst. Electr. Eng. 1969, 116, 457-465. [CrossRef] 